\title{
Application of Current Image-Enhanced Endoscopy in Gastric Diseases
}

\author{
Wansik Lee \\ Department of Internal Medicine, Chonnam National University Medical School, Hwasun, Korea
}

Image-enhanced endoscopy (IEE) plays an integral role in endoscopic diagnosis and treatment. IEE enables an early and accurate detection of cancer and characterization of lesions prior to therapeutic decisions. Ideal IEE can serve as an optical or digital chromoscopic endoscopy, as well as an optical biopsy that predicts exact histopathology. Several IEE modalities have recently been developed and are used in the clinical field. The stomach is a challenging organ for imaging because of its complex secretion function and status of Helicobacter pylori infection. Therefore, understanding the current IEE modalities for their clinical applicability in an evidence-based approach is warranted. Along with technology refinements, the new paradigm will be available for the diagnosis of gastric cancer or other conditions in the stomach in the near future. Clin Endosc 2021;54:477-487

Key Words: Diagnosis; Endoscopy; Neoplasm; Stomach

\section{INTRODUCTION}

The ultimate goal of performing endoscopy is to accurately identify the location and extent of the lesion and diagnose it in a minimally invasive way. Imaging-enhanced endoscopy (IEE) is an ideal new medical technology that satisfies these goals as a method to characterize lesions prior to biopsy and endoscopic treatment in real time. In the digestive tract, it is sometimes difficult to discriminate the surface microstructures, such as changes in microvascular and mucosal structures, which appear as chronic inflammatory changes, dysplasia, and malignancy from the gastric mucosa by conventional white light endoscopy (WLE). Indeed, accurate observation is essential for the early detection of cancer and differential diagnosis of precancerous lesions. However, the diagnosis of gastric disease can be misleading, and systematic imaging techniques should

Received: May 17, 2021 Revised: July 6, 2021

Accepted: July 8, 2021

Correspindence: Wansik Lee

Department of Internal Medicine, Chonnam National University Hwasun Hospital, 322 Seoyang-ro, Hwasun-eup, Hwasun-gun, Jeollanam-do 58128, Korea Tel: +82-61-379-7603, Fax: +82-62-379-7628, E-mail: jadelook@hanmail.net ORCID: https://orcid.org/0000-0002-8021-6228

It is the invited review article.

(c) This is an Open Access article distributed under the terms of the Creative Commons Attribution Non-Commercial License (http://creativecommons.org/ licenses/by-nc/3.0) which permits unrestricted non-commercial use, distribution, and reproduction in any medium, provided the original work is properly cited. be implemented to reduce the false diagnosis rate.

Therefore, IEE has been developed to maximize the contrast between the lesion and the surrounding mucosa using a simplistic method so that the lesion can be observed in detail, as in a chromoendoscopy. Among the imaging techniques of IEE, narrow band imaging (NBI) observes the lesion by separating only the visible light of a specific frequency with an optical filter from the existing white light. In addition, i-scan and fujinon intelligence chromoendoscopy (FICE) reconstruct the image using computer spectral estimation technology without using an optical filter. Recently, higher magnification techniques, such as confocal imaging and endocytoscopy that have a resolution power up to the cellular level, have been introduced in the clinical field to create new dimensions of the IEE field. In this review, the current application of IEE in the diagnosis and treatment of gastric diseases will be presented based on various imaging endoscopic techniques.

\section{ENDOSCOPIC IMAGING MODALITIES}

\section{Chromoendoscopy-Indigo carmine}

As a blue-colored agent, which does not react with or is absorbed by the mucosa, indigo carmine is useful for observing the pit pattern and structure by enhancing surface irregularities. It is generally diluted in physiological saline and used at a concentration of $0.2 \%(0.1 \%-0.4 \%)$. It is very useful for con- 
firming findings, such as irregular mucosal surfaces, depressed areas, and fusion deformities, as well as for diagnosing Barrett's esophagus, intestinal metaplasia, gastric adenoma, and gastric cancer. When used in combination with an IEE, the accuracy can be improved. In order to improve the quality of endoscopy, it is necessary to perform high-quality chromoendoscopy, if not IEE, and interpret the results based on a correct understanding of commonly used agents, such as indigo carmine.

\section{Magnifying endoscopy with NBI}

The basic principle of NBI is that when light is projected onto a tissue, the transmitted depth is proportional to the wavelength of the light. Since gastric cancer originates from the mucosa, it will be more helpful to observe microscopically if the short-wavelength blue light that can penetrate only the superficial mucosa is used. Because short-wavelength visible light is mainly absorbed by hemoglobin in blood vessels and is not reflected, it is observed in black. Therefore, when contrasting light is focused on a narrow area $(30 \mathrm{~nm})$ with wavelengths of $415 \pm 15 \mathrm{~nm}$ (blue) and $540 \pm 15 \mathrm{~nm}$ (green), subtle differences in mucosal lesions can be remarkably expressed in color and detailed images including blood vessels of the mucosal surface can be expressed.

Magnifying endoscopy (ME) allows the observation of the gastrointestinal mucosa at a high magnification of 80-120 times in real time during endoscopy. The microsurface structure and microvascular architecture of the mucosal surface can be observed in detail. If NBI is applied to ME, a clearer image of the microstructure of the gastric mucosa can be obtained. Therefore, ME with NBI (ME-NBI) is widely used for the diagnosis and discrimination of non-neoplastic and neoplastic lesions of the gastrointestinal tract and to confirm the extent of resection for therapeutic endoscopy.

After the development of NBI in 1999 and commercialization by Olympus in Japan, products of other companies using similar spectroscopy were introduced and are currently in use. In addition, the technology of implementing high-resolution images has also been developed in the recent years, and the widespread use of high-definition 1080i and 720p high-resolution image control technologies and high-resolution monitors have supported more detailed endoscopic examinations.

\section{3. i-SCAN}

i-scan is an improved software-based dynamic image enhancement technology developed by PENTAX, which provides an image of the mucosal surface structure and blood vessels. The image enhancement mode of the i-scan is surface enhancement (SE), contrast enhancement (CE), and tone enhancement (TE). SE emphasizes contrast based on the data obtained from each pixel and is a useful mode for the detec- tion and observation of lesions. CE can enhance the changes in the mucosal surface by adding a blue tint to the relatively dark area using the brightness data of the pixels. TE divides the image into red, green, and blue elements and then recombines a new image by transforming each element, emphasizing minute changes. The optical enhancement mode was recently developed and combines digital image processing using optical filters and narrow bands. This mode can reveal the surface structure in a clearer manner than the white-light endoscopes.

\section{FICE}

FICE is a spectrum-estimation technology developed by FUJINON. An improved image can be generated by extracting the image and arithmetically processing the image of a dedicated wavelength. FICE is also known as "multi-band image or optimal band image" because a variety of combinations of spectra can be selected. After extracting red, green, and blue images from the endoscopic image, FICE produces an improved image through arithmetic processing at a wavelength of 400-695 $\mathrm{nm}$. The principle of emphasizing blood vessels is similar to that of NBI, but instead of an optical filter, a spectrum estimation technique is used. Unlike NBI and blue light imaging (BLI)/ linked color imaging (LCI), i-scan and FICE are not images obtained by irradiating light of a specific wavelength but are images that are recreated through computer calculations from images obtained from WLE.

\section{BLI/LCI}

$\mathrm{BLI} / \mathrm{LCI}$ is a technology recently developed by Fujinon, and unlike NBI, which uses two wavelengths (blue $415 \mathrm{~nm}$ and green $540 \mathrm{~nm}$ ), it uses four wavelengths to create a specific image. BLI/LCI uses blue-violet wavelength, a short wavelength of $410 \mathrm{~nm}$, in addition to the existing red, green, and blue wavelengths to improve the distinction between the structure and boundaries of the lesion. LCI amplifies both blue-violet and white light wavelengths, so bright red becomes more vivid and the pale red becomes more pale, which helps in the detection of lesions. BLI amplifies the blue-violet wavelength to clearly distinguish the mucosal surface and vascular pattern and is similar to the image observed in NBI.

\section{Ultra-high magnification modalities}

Confocal laser endomicroscopy (CLE) is an advanced imaging modality that can obtain 1,000-fold magnification of the mucosal layer of the gastrointestinal tract in real time. CLE is based on tissue illumination using a low-power laser and the subsequent detection of fluorescent light that is reflected back from the tissue through a pinhole in the same focal plane. The high spatial resolution of CLE enables subsurface cellular imaging and detailed tissue architecture. 
Endocytoscopy is a contact light microscopy system integrated into the distal tip of a conventional endoscope. The latest endocytoscope is the fourth-generation and has an ultramagnification capability of over 500 times. It requires intraprocedural staining and enables on-site observation of not only structural atypia but also cytological atypia.

\section{OBSERVATION OF STOMACH WITH ME- NBI}

When the stomach is observed with a short-wavelength like $\mathrm{NBI}$, due to its wide lumen, it is difficult to observe the whole mucosa as a screening endoscopy. Therefore, NBI should be applied after the lesion is detected using a white light endoscope. According to a study of NBI without ME, intestinal metaplasia and adenoma can be diagnosed with sensitivity, specificity, positive predictive value, and negative predictive value of $71 \%, 58 \%, 65 \%$, and $66 \%$, respectively. These results are higher than those of $51 \%, 67 \%, 62 \%$, and $55 \%$ when using only WLE. ${ }^{2}$

However, unlike organs such as the esophagus and colon, the stomach with its wide luminal cavity is not easy to observe with NBI due to a dark light source, and the image quality is coarse and dark. Until now, the usefulness of stomach observation has not been verified with NBI alone, and NBI is useful only when used in conjunction with ME. ME-NBI can clearly observe microsurface as well as microvascular patterns, while WLE can usually detect only partial microvascular structures. When the gastric mucosa is observed with ME, the main microvascular structure is the subepithelial capillary network (SECN), collecting venules, and microvessels. The microscopic surface structure of the mucous membrane is the marginal crypt epithelium, crypt opening, or gastric pit. ${ }^{3}$

\section{Chronic gastritis}

In the case of Helicobacter pylori (H. pylori) infection, observation of the gastric pit and vascular pattern through ME-NBI of the gastric body is useful. The main findings suggesting $H$. pylori infection are: 1) loss or irregularity of the collecting venule, 2) expansion of the unclear or irregular gastric pit, and 3) A meta-analysis loss of normal SECN (Fig. 1). Meta-analysis including six studies showed that ME-NBI predicted $H$. pylori infection with 96\% (95\% confidence interval [CI], 94\%-97\%) sensitivity, 91\% (95\% CI, 87\%-93\%) specificity, and when a receiver operator characteristic curve was created, the area under the curve (AUC) value was expanded to 0.987. Thus, the usefulness of the endoscope was confirmed by ME-NBI, ${ }^{2}$ and the differentiating degree of sensitivity, specificity, positive predictive value, and negative predictive value were reported as $100 \%, 92.7 \%, 83.8 \%$, and $100 \%$, respectively. ${ }^{4}$

The selection of patients with a high risk of gastric cancer was emphasized based on the progression of gastritis and intestinal metaplasia. To this end, a more accurate evaluation was recommended using IEE including ME-NBI at MAPS II (management of epithelial precancerous conditions and lesions in the stomach). ${ }^{5}$ In MAPS II normal SECN and round pit are lost, and an array of amorphous collective venules appears, and the mucosa of the upper body changes to a ridged, papillary, or villous pattern similar to that of the antrum. Intestinal metaplasia was found in ME-NBI as a characteristic finding called the light blue crest (LBC); this finding was histologically associated with intestinal metaplasia representing high specificity finding. ${ }^{6,7} \mathrm{LBC}$ is a thin, blue fluorescent ray that appears along the ridge of the microscopic surface of the gastric mucosa. It is presumed to be a reflection of the short-wavelength

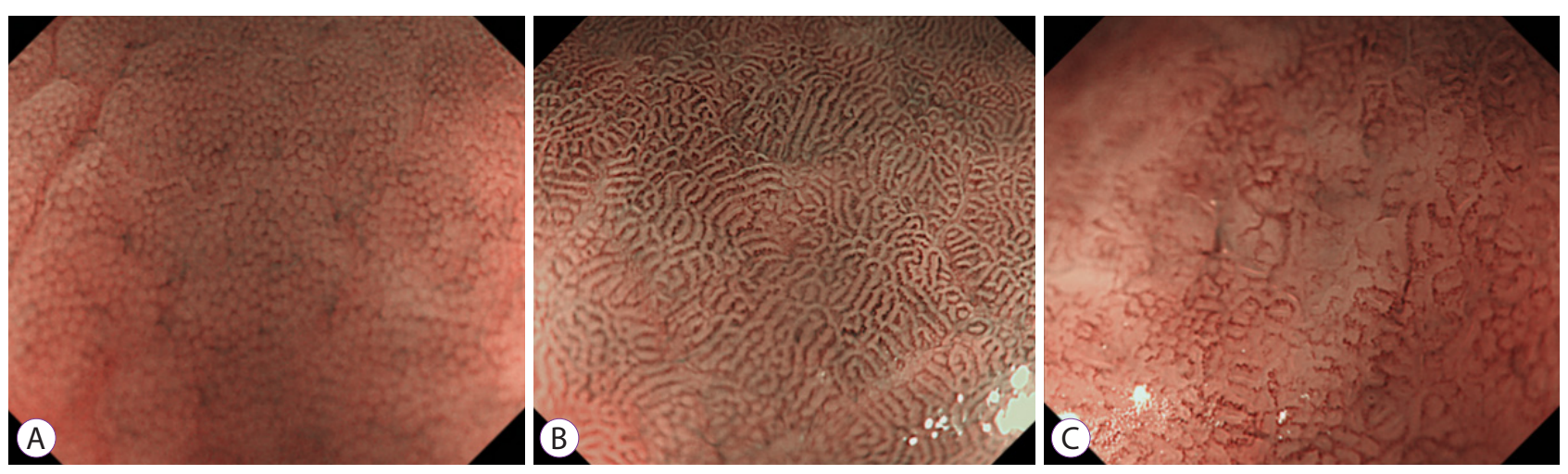

Fig. 1. ME-NBI findings of stomach mucosa according to location and Heliciobacter pylori infection status. (A) Fundal mucosa without $H$. pylori infection. (B) Antral mucosa without $H$. pylori infection. (C) Fundal mucosa with $H$. pylori infection. ME-NBI, magnifying endoscopy with narrow band imaging. 
visible rays on the brush border (Fig. 2). Along with LBC, another finding, white opaque substance (WOS), is a white substance that exists in the mucosal surface and interferes with the observation of sub-epithelial microvessels. It is known as a phenomenon in which the light source from the endoscope is reflected or dispersed in small droplets of fat located in the epithelium, causing difficulty in observing the blood vessels. It is mainly observed in gastric dysplasia and gastric cancer lesions, as well as in intestinal metaplasia (Fig. 3). ${ }^{7-9}$

\section{Gastric adenoma}

When an elevated gastric adenoma is observed with ME, microvessels are rarely observed, resulting in a lack of discriminating power with the surrounding mucosa. According to a recent study, ME-NBI observed a characteristic "WOS" in an elevated gastric adenoma. When 46 cases of elevated adenoma and cancer were analyzed, a typical regular WOS was observed in $100 \%$ of gastric adenomas and irregularly shaped WOS in $83 \%$ of gastric cancer cases. Regular type WOS was reported as a characteristic finding of gastric adenoma with ME-NBI. ${ }^{10}$ Overall, ME-NBI findings of gastric adenoma are irregular microsurface and microvascular structures, but relative regularity is maintained compared to gastric cancer, and WOS is better observed than gastric cancer. ${ }^{9}$

\section{Early gastric cancer (EGC)}

ME-NBI is considered an effective method for predicting the degree of differentiation of gastric cancer, defining margins, and predicting the depth of invasion. In one study, depressed well-differentiated adenocarcinoma (intestinal type) showed loss of SPEC and appearance of a demarcation line with cancer and irregular microvessels. ${ }^{8}$ While in undifferen-
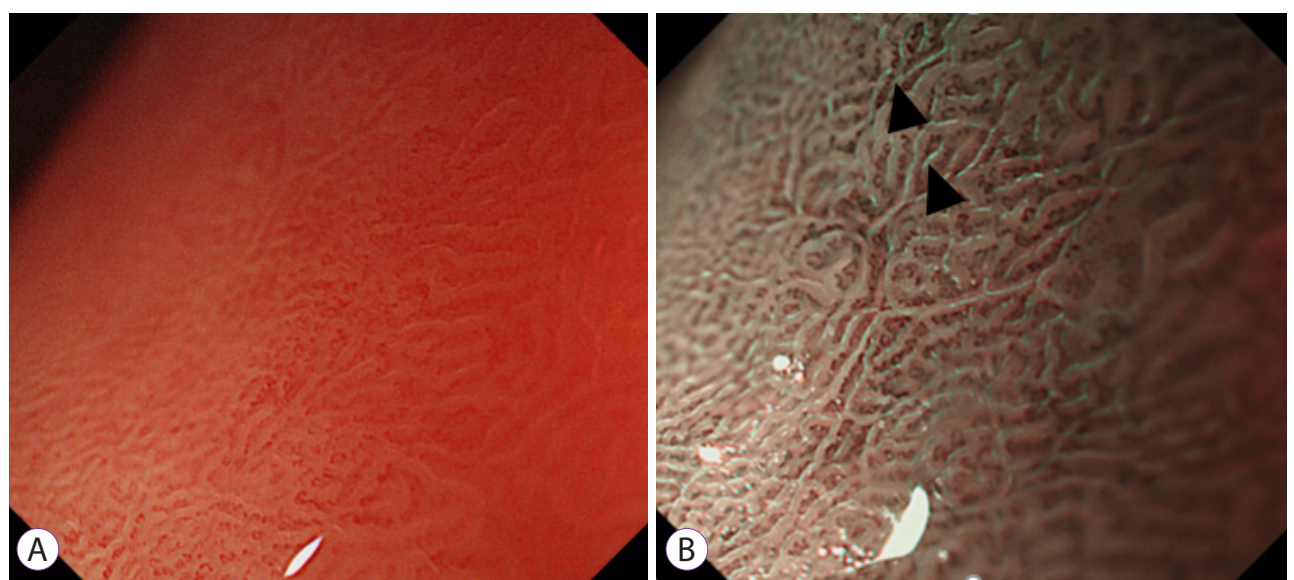

Fig. 2. ME-NBI finding of intestinal metaplasia featuring light blue crest. (A) White light endoscopy. (B) ME-NBI. ME-NBI, magnifying endoscopy with narrow band imaging.
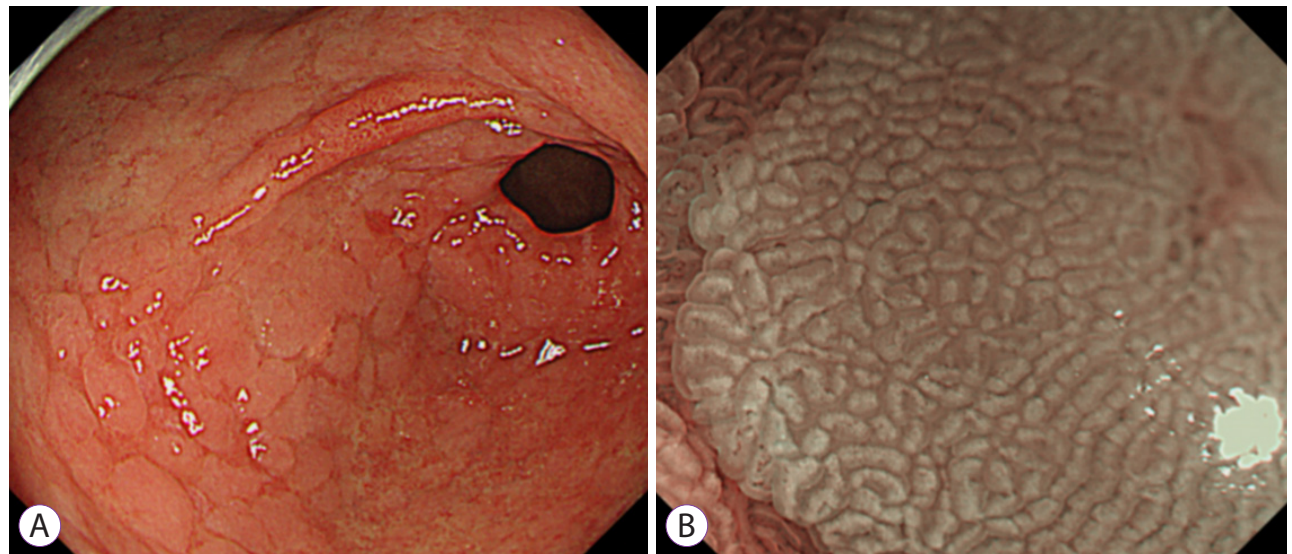

Fig. 3. ME-NBI finding in intestinal metaplasia featuring white opaque substance. (A) White light endoscopy. (B) ME-NBI. ME-NBI, magnifying endoscopy with narrow band imaging. 
tiated adenocarcinoma (diffuse type), microvessels were reduced. However, other studies have reported that demarcation lines are also observed in undifferentiated adenocarcinoma. They argued that the degree of differentiation could not be predicted using the demarcation line alone. In another study on the microvascular pattern, $68.4 \%$ of well-differentiated adenocarcinomas had microvessels in a fine network pattern, and 85.3\% of undifferentiated adenocarcinoma had a corkscrew pattern. ${ }^{11}$

In a study on the boundary of EGC, ME-NBI was compared with indigo carmine chromoendoscopy to determine whether the boundary of gastric cancer could be clearly observed. The margin of EGC could be observed more accurately with MENBI than with indigo carmine chromoendoscopy ( $97.4 \%$ vs. $77.8 \%) .{ }^{12}$ In a recent study on the prediction of the depth of invasion, microvessel patterns in EGC were classified into three patterns: mesh type, loop type, and interrupted type. The mesh type was observed as a circular pit-pattern at $88.9 \%$, and the loop type was observed in a non-circular pit pattern at
$100 \%$. In cases showing both mesh type and loop type, $94.9 \%$ of cancer was confined to the mucosa, and $92.3 \%$ of the interrupted type showed submucosal layer invasion. ${ }^{13}$

As in the aforementioned studies, ME-NBI is useful for diagnosing EGC. In clinical practice, however, due to the discrepancy among observers for the analysis of microvessels and pit morphology, the usefulness of ME-NBI is somewhat limited. In addition, a classification method for the discrimination of neoplastic lesions in the stomach has not yet been established. Yao et al. presented 'VS' system according to the microvascular morphology (V) and microsurface structure (S), which is well-studied. ${ }^{14}$ Currently, the VS classification system is the simplest and most sensitive diagnostic method for EGC. According to this system, the existence of an irregular microvascular pattern with a demarcation line or an irregular microsurface pattern with demarcation line is defined as the criteria suitable for the diagnosis of gastric cancer, and theoretically, $95 \%$ of EGCs can be diagnosed based on these criteria. ${ }^{15}$ The demarcation line refers to the visible line between the lesion
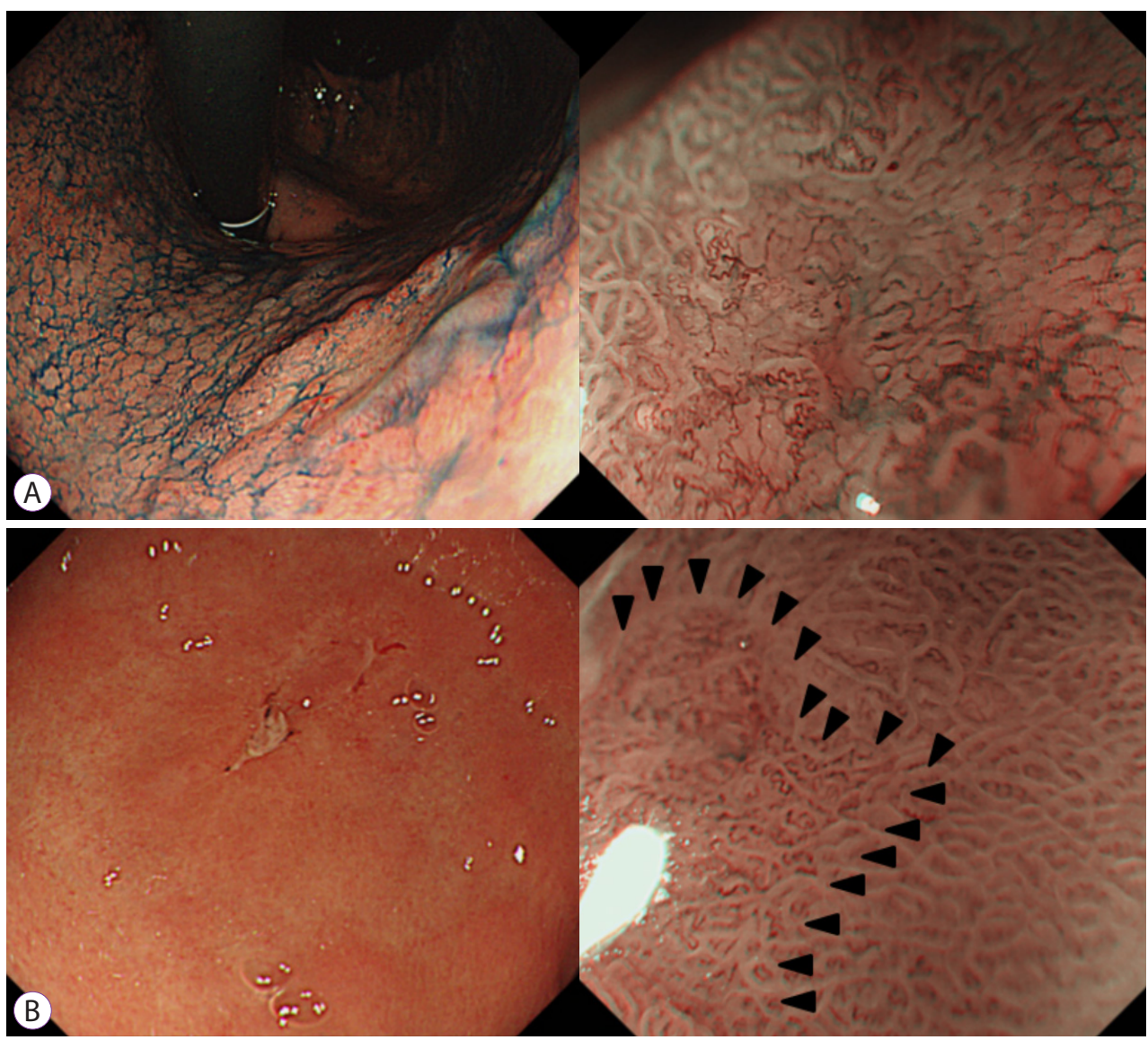

Fig. 4. ME-NBI findings on gastric adenocarcinoma featuring demarcation line and irregular microvascular or microsurface pattern. (A) Well differentiated type. (B) Signet ring cell type EGC. ME-NBI, magnifying endoscopy with narrow band imaging; EGC, early gastric cancer. 
and the normal mucosa where the shape of the microscopic surface changes abruptly (Fig. 4).

The findings suggesting EGCs in the VS classification are irregular microsurface morphology and/or microvascular shape along with distinct borders to the surrounding mucosa. In a randomized controlled study, the accuracy of ME-NBI using the VS classification was $90.4 \%$ in determining gastric cancer for depressive lesions of $10 \mathrm{~mm}$, which is superior to that of a general WLE, and it could be cost effective by reducing the number of tissue biopsies. ${ }^{16,17}$ In contrast, in one meta-analysis, the sensitivity of diagnosing cancer using the VS system for depressed lesions was $64 \%$, which was significantly lower than that of $90 \%$ of studies using other classification methods. ${ }^{18}$ This means that the VS classification obscurely defines microvascular irregularities and is sometimes difficult to diagnose accurately depending on the skill level of the endoscopist. There is a need for a classification system with a higher degree of agreement between observers and easier to acquire and interpret the endoscopic findings through further studies.

In elevated EGC, the microvascular pattern is often obscured by WOS; therefore, only microscopic surface patterns may be useful for diagnoses. WOS appears in chronic atrophic gastritis with intestinal metaplasia or intestinal-type gastric adenocarcinoma. ${ }^{19}$ Different manifestations of WOS can help differentiate between cancer and adenoma. While differentiated-type adenocarcinoma shows an irregularly shaped WOS, adenoma is observed to feature regular WOS and undifferentiated-type adenocarcinoma does not feature WOS.

In other studies, using criteria including the loss of microsurface structures, microvascular dilation, and irregularity, ME-NBI was found to show a high sensitivity in depressed or flat EGCs compared with WLE. ${ }^{20}$ For gastric adenoma and differentiated-type gastric cancer, a five-subtype classification system based on microvascular and microsurface patterns was proposed. Lesions with clear or unclear microvascular and clear microsurface patterns can be diagnosed as gastric adenoma, and other subtypes can be diagnosed as differentiated-type gastric adenocarcinoma. ${ }^{21}$ In addition, a white globe appearance, a small spherical white change $<1 \mathrm{~mm}$ on MENBI corresponding to necrotizing tissue in an enlarged neoplastic gland has been presented as a characteristic finding in differentiated-type gastric cancer. ${ }^{22}$

\section{CLINICAL APPLICATION OF ME-NBI IN GASTRIC CANCER}

There has been an international expert consensus on a simple diagnostic algorithm for gastric cancer, MESDA-G. ${ }^{23}$ WLE is used for the overall observation of the stomach, and if a suspected lesion is found, then ME is performed to diagnose cancer. At first, when the demarcation line is observed, a cancerous lesion is suspected, and if not, a non-cancerous lesion is suspected. Subsequently, if any of the two factors, including the microvessel and microsurface pattern are judged to be irregular, the cancer is diagnosed.

\section{Usefulness for the classification of EGC}

There is little clinical evidence for the usefulness of an MENBI for assessing the depth of invasion of gastric adenocarcinoma. In 2010, a consensus report on NBI concluded that NBI is not reliable for predicting the depth of invasion of gastric cancer. However, various data have been presented for the comparison of ME-NBI with the histologic classification of gastric adenocarcinoma. According to the vascular pattern, the fine network pattern is a characteristic of differentiated-type gastric cancer, while undiffrentiated-type cancer features have a corkscrew pattern. ${ }^{24}$ However, flat or depressed, pale colored undifferntiated-type EGC often exhibits normal microvascular and microscopic patterns, making an NBI diagnosis difficult.

In other studies, loop morphology in lobules (intralobular loop [ILL]) was used to perform a new classification. ILL-1 is characterized by a loop-like microvessels located within the villi surface structure, and ILL-2 is a form in which the villi structure begins to be destroyed. ${ }^{25}$ Lesions with both fine network morphology and ILL-1 morphology are considered as differentiated-type gastric cancer, whereas lesions with a spiral structure are likely to be undifferentiated-type gastric cancer. The difference in the NBI findings on well-differentiated EGC is related to the mucin phenotype of the lesion. Most lesions showing the ILL form are gastric or gastrointestinal phenotypes, whereas microscopic network-shaped lesions are mainly of the intestinal phenotype. ${ }^{26}$

Some undifferentiated type of EGC usually progresses from the deep layer to the surface layer. The S-type with irregular superficial type and V-type with irregular microvascular type in which the surface microstructure has disappeared are correla are correlated with the development period of undifferentiated-type cancer, and the M-type (mixed type) is correlated with the progression of a histopathology of undifferentiated-type EGC. ${ }^{27}$

\section{Usefulness in evaluation of lesion boundary}

As endoscopic resection for EGC has been widely performed, an accurate evaluation of the lesion, especially margin clarification, has become very important. It is difficult to accurately determine the boundary in $20 \%$ of EGCs with magnification alone. In more than $70 \%$ of these uncertain boundaries, the accuracy can be improved with ME-NBI, and a randomized study comparing with conventional chromoen- 
doscopy showed a significantly higher accuracy in evaluating the boundaries of the lesion. ${ }^{13}$

The VS classification system has proven to be very useful for delineating the margin of EGC. According to VS classification, the margin of the lesion appears to be a cancer-specific demarcation between irregular microvessels or microsurface regions and regular surroundings. It is difficult to evaluate the margin of undifferentiated-type adenocarcinoma due to diffuse, uneven lateral progression within the mucosa causing an obscured demarcation line. ${ }^{28}$ There is suggestion that in using the VS classification, the boundary is appropriately evaluated even in undifferentiated-tyoe EGC. ${ }^{29}$ In clinical practice, confirming the margin by biopsy of the surrounding mucosa may be necessary.

\section{Gastric lymphoma}

Gastric mucosa-associated lymphoid tissue (MALT) lymphoma is occasionally difficult to differentiate from EGC. MENBI is helpful in observing irregular blood vessels and crypt epithelium swelling, especially in the shape of a tree trunk with long, bare branches. ${ }^{30}$ However, these findings are also used in gastric adenocarcinoma with poor differentiation. Therefore, it may be useful to perform additive definitive target biopsy to assess the complete remission after treatment. ${ }^{31}$

\section{Usefulness in evaluation of the lesion after treatment}

There have been reports on the usefulness of ME-NBI in predicting the outcome after $\mathrm{H}$. pylori eradication, to evaluate the remission of gastric MALT lymphoma, or as a method to detect recurrence after endoscopic resection. In the case of the successful eradication of $H$. pylori, the elongated and enlarged pit pattern turned into a small, round shape. However, when accompanied by severe atrophy or intestinal metaplasia, these changes are not well-observed. ${ }^{32}$

According to a study that analyzed the findings of ME-NBI after remission of gastric MALT lymphoma, the microstructure and subepithelial capillary networks were restored and abnormal vessels disappeared; hence, tissue biopsy could be avoided, and it might have been possible to assess the response to treatment or to perform ME-NBI-guided targeted biopsies. ${ }^{33}$ Also, in the follow-up after endoscopic resection for EGC, there was a correlation between healed ulcer scars and local recurrences in the case of microstructural destruction. They argued that ME-NBI is the most effective method for predicting local recurrences during follow-up endoscopy. ${ }^{34}$

\section{FICE AND I-SCAN IN GASTRIC DISEASE}

FICE is reported to display high accuracy in observing changes in intestinal metaplasia. Additionally, in conjunction with magnification, an accuracy of over $85 \%$ is achieved especially when a "long large crest" was observed. ${ }^{35}$ FICE increased the color contrast between cancerous and benign mucosal lesions to facilitate discrimination; moreover, this diagnostic ability is more useful in depressed EGC. If the i-scan is applied with a magnification, it can be used to assess the microstructure of the gastric mucosa, which helps to predict the presence of $H$. pylori infection. Likewise, both the FICE and i-scan can be used to determine the horizontal extent of gastric cancer due to an increased contrast when compared to standard WLE. ${ }^{36}$ Taken together, unlike NBI the clinical evidence is relatively insufficient; therefore, a reliable classification system for diagnosing cancer has not been established.

\section{LCI AND BLI IN GASTRIC DISEASES}

The LCI and BLI mode is a recently developed key technology for the diagnosis of gastric cancer in screening endoscopy. The contrast between the mucosa and blood vessels was clear, and the ability to detect lesions in the far-field was better in these modes. The zoom function in BLI-bright images showed higher accuracy than WLE in diagnosing gastric adenoma. In the evaluation using LCI, the majority of the EGCs appeared orange. LCI shows a distinct color difference from the normal mucosa or intestinal metaplasia. This facilitates the detection of diffuse flat EGC, especially metachronous lesions that occur after endoscopic resection.

Intestinal metaplasia manifests in the form of purple and green spots at the LCI, and during endoscopic screening, these features are clinically important because of the large color contrast with gastric cancer. In addition, LCI makes it possible to rule out cancer even when the gross finding suggests cancer with WLE emphasizing subtle color tone changes preceding morphological changes. ${ }^{37}$

\section{CLE}

\section{Principle and method}

CLE uses a confocal laser as a light source to manifest a magnified image of 1,000 times by obtaining only a fluorescence image of a specific wavelength through a reflector. 'Confocal' refers to the state in which the illumination and detection planes exist in one plane. To date, two types of CLEs have been clinically studied: one was integrated with a white optical endoscope (endoscope-based CLE [eCLE]; Pentax, Tokyo, Japan) and the other was the probe type developed by Cellvizio (probe-based CLE [pCLE]; Mauna Kea Technologies, Paris, 
France). When the $488 \mathrm{~nm}$ laser is released, it penetrates up to $250 \mu \mathrm{m}$ from the mucosal surface and then returns with a wavelength of $505 \mathrm{~nm}$ to $585 \mathrm{~nm}$ of the fluorescent substance injected into the vein to finally obtain a high-resolution black and white cross-sectional image. ${ }^{38}$ In order to obtain a high-resolution image through CLE based on this principle, an additional fluorescent contrast agent is required. Typical examples are fluorescein and acriflavine. Fluorescein (10\%) is administered intravenously, and spread to the cell tissues, excluding the cell nucleus, and observing the tissue structure and blood vessels to the deep mucosa layer of $250 \mu \mathrm{m}$ can be possible.

\section{Clinical application of CLE in the stomach}

CLE can detect typical goblet cells, and prominent villi and intestinal metaplasia can be diagnosed relatively easily. Therefore, in most studies, CLE target biopsy has a higher rate of diagnosis in intestinal metaplasia than standard biopsy through WLE. The sensitivity and specificity of pCLE in the diagnosis of intestinal metaplasia were 92\% (90\%-94\%) and 97\% (96\%-98\%). ${ }^{39}$ Additionally, recent studies have shown that CLE has a sensitivity and specificity of $81 \%$ and $98 \%$, respectively, in precancerous lesions and $91 \%$ and $99 \%$ in gastric cancer, respectively, showing high diagnostic value (Fig. $5){ }^{40}$ In a meta-analysis of 23 single-center studies conducted in Asia (China, Japan, Korea, Singapore, and Thailand), CLE diagnosed gastric cancer with a sensitivity and specificity of 91\% (88\%-94\%) and 99\% (99\%-99\%), respectively. A subtype analysis found a sensitivity and specificity of $88 \%(80 \%-94 \%)$ and $99 \%(99 \%-100 \%)$ in undifferentiated-type gastric cancer and $87 \%(80 \%-92 \%)$ and $99 \%(99 \%-100 \%)$ in differentiated-type gastric cancer. ${ }^{39}$

Although there are no reports directly comparing CLE to $\mathrm{NBI}$ or FICE yet, CLE reduced the average number of biopsies performed per patient. Considering the ulcers and damage to the gastric mucosa and the time and cost of the biopsy proce-
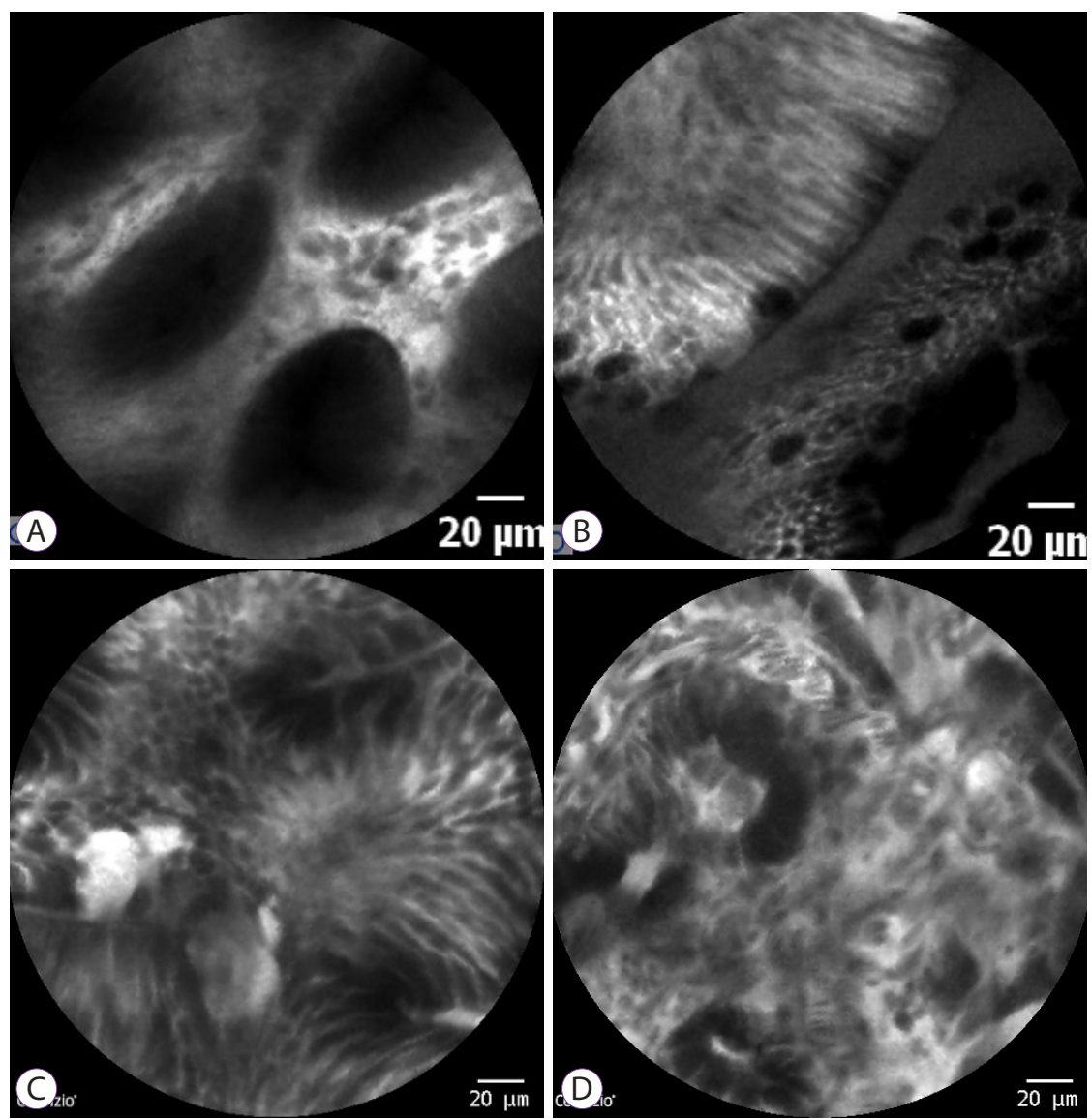

Fig. 5. CLE image of various gastric lesions. (A) Normal epithelium, featuring regularly arranged glands and epithelial cells with homogenous size and height. (B) Intestinal metaplasia with typical blackish goblet cells and prominent villi. (C) High-grade dysplasia featuring irregular glandular structure and increased epithelial cellularity. (D) Gastric cancer showing complete destruction of glandular structure, irregular shape and size of tumor cells, and disarrayed vasculature. CLE, confocal laser endomicroscopy. 
dure, these optical methods can complement the shortcomings of an existing biopsy-based diagnosis alone. In addition, the real-time use of CLE can help in endoscopic treatment. During endoscopic submucosal dissection (ESD), CLE can increase the diagnostic accuracy of the lesion and secure the resection margin. In a prospective randomized study of 101 patients undergoing ESD, pCLE could specify the resection margin more effectively. The ratio of less than $1 \mathrm{~mm}$ in distance was higher in the pCLE group than in the WLE group, especially in flat type EGC, although there was no difference in the rate of complete resection between the two groups. ${ }^{41}$ In another study, CLE can secure additional cancer cells in the biopsy ( $65 \%$ vs. $30 \%, p=0.002$ ) than WLE alone, indicating the possibility of facilitating the biopsy efficiency. ${ }^{42}$

\section{ENDOCYTOSCOPY}

\section{Principle and method}

Endocytoscopy is an ultra-high magnification endoscopy technique that enables the microscopic examination of the mucosal surface by staining with an endoscope. First-generation endocytosopy was first developed in 2003, and the latest fourth-generation endoscope has a magnification of 500 times that can visualize cell nuclei. ${ }^{43,44}$ The 4 th generation endoscopy (GIF-H290EC; Olympus Medical Systems Corp., Tokyo, Japan) can gradually expand up to 500 times and has an outer diameter of $9.7 \mathrm{~mm}$, and a range of $570 \mu \mathrm{m} \times 500 \mu \mathrm{m}$ can be observed.

In the stomach, a normal mucosa features with glands of a uniform shape and regularly arranged nuclei, while in cancer, cellular and glandular structures are not maintained, and darkly stained, irregular variable-sized nuclei with edema are observed. This "enlarged nuclear sign" is characteristic and is helpful in the diagnosis of gastric cancer. In gastric adenoma, despite the structural deformity, the glandular structure remains identifiable, and the nucleus is small, round, and poorly stained.

\section{Clinical application of endocytoscopy in the stomach}

Compared with the esophagus, there is a limitation in obtaining adequate images due to the functions of absorption and secretion of the gastric mucosal epithelium. ${ }^{45,46}$ To overcome this limitation, a study was conducted to improve staining by using mucolytic pronase and a solution containing dimethicone was used prior to the procedure, and high-quality images were obtained in more than $80 \%$ of the subjects. ${ }^{47}$

Studies have shown that the normal gastric mucosa ob- served by endocytoscopy has a regular gland structure with a smooth surface and is characterized by the appearance of a rim, well-preserved glandular lumen, and small, uniformly sized nuclei. ${ }^{48}$ Hyperplastic polyps have a larger star-shaped glandular lumen and small, regular nuclei. ${ }^{49}$ Notably, H. pylori as a moving rod-shaped bacterium was observed through an ex vivo endocytoscopy study. ${ }^{50}$ Whether it is possible to observe $H$. pylori in a moving form in vivo needs further study for the purpose of checking $H$. pylori infection in real time.

A Japanese study conducted in vivo endocytoscopy of 26 patients with differentiated-type adenocarcinoma and four patients with undifferentiated-type adenocarcinoma. Differentiated-type adenocarcinoma exhibited irregularly branched glands, glandular lumen of various widths, irregularly arranged epithelium, and strongly stained nuclei; while in undifferentiated-type adenocarcinoma, the tubular structure was lost and the nucleus was overstained and in heterogeneous shape. These findings coincided with the microscopic histological results after biopsy (approximately $90 \%$ ). ${ }^{51}$ The fact that the in vivo endocytoscopic image was similar to the histopathologic image suggests that endocytoscopy has a higher potential in the diagnosis of gastric cancers and is expected to serve as the foundation for the role of optical biopsy. According to a recent study, the accuracy of the diagnosis of EGC was confirmed to have a sensitivity of $97.0 \%-91.3 \%$, a specificity of $75.0 \%-80.0 \%$, and an accuracy of $83.7 \%$. Excluding adenomas, the distinction between cancer and benign lesions was comparable to that confirmed by conventional histopathology. ${ }^{47}$ In particular, the enlarged nuclear sign helps to increase the diagnostic accuracy by distinguishing gastric cancer from benign lesions, and these characteristic findings are considered to be helpful in actual clinical applications. ${ }^{47}$

\section{CONCLUSIONS}

Several imaging methods have been devised and used to overcome the diagnostic indistinctiveness of the stomach. Conventional WLE with or without chromoendoscopy with indigo carmine is still a cardinal screening method for detecting EGC. However, to accurately define the lesion and interpret the histological characteristics, IEE is presumed to be essential in current clinical practice. ME was a paradigm shift in the field of IEE in that it made it possible to classify the microsurface and vascular pattern for the exact prediction of the corresponding histopathology. ME-NBI or other optical imaging techniques help to detect and diagnose various gastric lesions with optical biopsy-like precision, if applied with a clear and experienced protocol. Ultra-high magnification en- 
doscopy is clinically available and now opens new possibilities for real-time histologic examination.

It takes abundant effort, experience, and time to observe and analyze the microstructures and microvessels. Overcoming the inconsistency of diagnoses between observers is a task to be overcome in the future. To achieve this, a more defining imaging technology in conjunction with a detailed unified classification system will be necessary. Further research needs to be performed in order for IEE to play a role as an electronic chromoendoscopy, enabling optical biopsy capacity for accurately diagnosing the lesion to benefit therapeutic outcomes in clinical practicality.

\section{Conflicts of Interest}

The author has no potential conflicts of interest.

Funding

None.

ORCID

Wansik Lee:

https://orcid.org/0000-0002-8021-6228

\section{REFERENCES}

1. Muto M, Katada C, Sano Y, Yoshida S. Narrow band imaging: a new diagnostic approach to visualize angiogenesis in superficial neoplasia. Clin Gastroenterol Hepatol 2005;3:S16-S20.

2. Capelle LG, Haringsma J, de Vries AC, et al. Narrow band imaging for the detection of gastric intestinal metaplasia and dysplasia during surveillance endoscopy. Dig Dis Sci 2010;55:3442-3448.

3. Yao K. The endoscopic diagnosis of early gastric cancer. Ann Gastroenterol 2013;26:11-22.

4. Qi Q, Guo C, Ji R, Li Z, Zuo X, Li Y. Diagnostic performance of magnifying endoscopy for Helicobacter pylori infection: a meta-analysis. PLoS One 2016;11:e0168201.

5. Anagnostopoulos GK, Yao K, Kaye P, et al. High-resolution magnification endoscopy can reliably identify normal gastric mucosa, Helicobacter pylori-associated gastritis, and gastric atrophy. Endoscopy 2007;39:202-207.

6. Pimentel-Nunes P, Libânio D, Marcos-Pinto R, et al. Management of epithelial precancerous conditions and lesions in the stomach (MAPS II): European Society of Gastrointestinal Endoscopy (ESGE), European Helicobacter and Microbiota Study Group (EHMSG), European Society of Pathology (ESP), and Sociedade Portuguesa de Endoscopia Digestiva (SPED) guideline update 2019. Endoscopy 2019;51:365-388.

7. Uedo $\mathrm{N}$, Ishihara $\mathrm{R}$, Iishi $\mathrm{H}$, et al. A new method of diagnosing gastric intestinal metaplasia: narrow-band imaging with magnifying endoscopy. Endoscopy 2006;38:819-824.

8. Pimentel-Nunes P, Dinis-Ribeiro M, Soares JB, et al. A multicenter validation of an endoscopic classification with narrow band imaging for gastric precancerous and cancerous lesions. Endoscopy 2012;44:236246.

9. Yao K, Iwashita A, Tanabe H, et al. White opaque substance within superficial elevated gastric neoplasia as visualized by magnification endoscopy with narrow-band imaging: a new optical sign for differentiating between adenoma and carcinoma. Gastrointest Endosc 2008;68:574580 .
10. Matsushita M, Mori S, Uchida K, Nishio A, Okazaki K. "White opaque substance" and "light blue crest" within gastric flat tumors or intestinal metaplasia: same or different signs? Gastrointest Endosc 2009;70:402; author reply 402-403.

11. Yao K, Oishi T. Microgastroscopic findings of mucosal microvascular architecture as visualized by magnifying endoscopy. Dig Endosc 2001;13:S27-S33.

12. Nakayoshi T, Tajiri H, Matsuda K, Kaise M, Ikegami M, Sasaki H. Magnifying endoscopy combined with narrow band imaging system for early gastric cancer: correlation of vascular pattern with histopathology (including video). Endoscopy 2004;36:1080-1084.

13. Kiyotoki S, Nishikawa J, Satake M, et al. Usefulness of magnifying endoscopy with narrow-band imaging for determining gastric tumor margin. J Gastroenterol Hepatol 2010;25:1636-1641.

14. Yagi K, Nakamura A, Sekine A, Umezu H. Magnifying endoscopy with narrow band imaging for early differentiated gastric adenocarcinoma. Dig Endosc 2008;20:115-122.

15. Yao K, Anagnostopoulos GK, Ragunath K. Magnifying endoscopy for diagnosing and delineating early gastric cancer. Endoscopy 2009;41:462467.

16. Yao K. Clinical application of magnifying endoscopy with narrow-band imaging in the stomach. Clin Endosc 2015;48:481-490.

17. Ezoe Y, Muto M, Uedo N, et al. Magnifying narrowband imaging is more accurate than conventional white-light imaging in diagnosis of gastric mucosal cancer. Gastroenterology 2011;141:2017-2025.e3.

18. Hu YY, Lian QW, Lin ZH, Zhong J, Xue M, Wang LJ. Diagnostic performance of magnifying narrow-band imaging for early gastric cancer: A meta-analysis. World J Gastroenterol 2015;21:7884-7894.

19. Yao K, Iwashita A, Nambu M, et al. Nature of white opaque substance in gastric epithelial neoplasia as visualized by magnifying endoscopy with narrow-band imaging. Dig Endosc 2012;24:419-425.

20. Kaise M, Kato M, Urashima M, et al. Magnifying endoscopy combined with narrow-band imaging for differential diagnosis of superficial depressed gastric lesions. Endoscopy 2009;41:310-315.

21. Nonaka K, Arai S, Ban S, et al. Prospective study of the evaluation of the usefulness of tumor typing by narrow band imaging for the differential diagnosis of gastric adenoma and well-differentiated adenocarcinoma. Dig Endosc 2011;23:146-152.

22. Doyama H, Yoshida N, Tsuyama S, et al. The "white globe appearance" (WGA): a novel marker for a correct diagnosis of early gastric cancer by magnifying endoscopy with narrow-band imaging (M-NBI). Endosc Int Open 2015;3:E120-E124.

23. Muto M, Yao K, Kaise M, et al. Magnifying endoscopy simple diagnostic algorithm for early gastric cancer (MESDA-G). Dig Endosc 2016;28:379393.

24. Nakayoshi T, Tajiri H, Matsuda K, Kaise M, Ikegami M, Sasaki H. Magnifying endoscopy combined with narrow band imaging system for early gastric cancer: correlation of vascular pattern with histopathology (including video). Endoscopy 2004;36:1080-1084.

25. Yokoyama A, Inoue $\mathrm{H}$, Minami $\mathrm{H}$, et al. Novel narrow-band imaging magnifying endoscopic classification for early gastric cancer. Dig Liver Dis 2010;42:704-708.

26. Kobayashi M, Takeuchi M, Ajioka Y, et al. Mucin phenotype and narrow-band imaging with magnifying endoscopy for differentiated-type mucosal gastric cancer. J Gastroenterol 2011;46:1064-1070.

27. Li HY, Ge ZZ, Fujishiro M, Li XB. Current clinical applications of magnifying endoscopy with narrow band imaging in the stomach. Diagn Ther Endosc 2012;2012:271914.

28. Nagahama T, Yao K, Maki S, et al. Usefulness of magnifying endoscopy with narrow-band imaging for determining the horizontal extent of early gastric cancer when there is an unclear margin by chromoendoscopy (with video). Gastrointest Endosc 2011;74:1259-1267.

29. Okada K, Fujisaki J, Kasuga A, et al. Diagnosis of undifferentiated type early gastric cancers by magnification endoscopy with narrow-band im- 
aging. J Gastroenterol Hepatol 2011;26:1262-1269.

30. Nonaka K, Ishikawa K, Shimizu M, et al. Education and imaging. Gastrointestinal: gastric mucosa-associated lymphoma presented with unique vascular features on magnified endoscopy combined with narrow-band imaging. J Gastroenterol Hepatol 2009;24:1697.

31. Ono $\mathrm{S}$, Kato M, Ono Y, et al. Target biopsy using magnifying endoscopy in clinical management of gastric mucosa-associated lymphoid tissue lymphoma. J Gastroenterol Hepatol 2011;26:1133-1138.

32. Okubo M, Tahara T, Shibata T, et al. Changes in gastric mucosal patterns seen by magnifying NBI during $\mathrm{H}$. pylori eradication. J Gastroenterol 2011;46:175-182.

33. Ono S, Kato M, Ono Y, et al. Characteristics of magnified endoscopic images of gastric extranodal marginal zone B-cell lymphoma of the mucosa-associated lymphoid tissue, including changes after treatment. Gastrointest Endosc 2008;68:624-631.

34. Lee TH, Chung IK, Park JY, et al. Usefulness of magnifying endoscopy in post-endoscopic resection scar for early gastric neoplasm: a prospective short-term follow-up endoscopy study. World J Gastroenterol 2009;15:349-355.

35. Osawa H, Yamamoto H. Present and future status of flexible spectral imaging color enhancement and blue laser imaging technology. Dig Endosc 2014;26 Suppl 1:105-115.

36. Jang JY. The past, present, and future of image-enhanced endoscopy. Clin Endosc 2015;48:466-475.

37. Osawa H, Miura Y, Takezawa T, et al. Linked color imaging and blue laser imaging for upper gastrointestinal screening. Clin Endosc 2018;51:513-526.

38. Jeon SR, Cho WY, Jin SY, Cheon YK, Choi SR, Cho JY. Optical biopsies by confocal endomicroscopy prevent additive endoscopic biopsies before endoscopic submucosal dissection in gastric epithelial neoplasias: a prospective, comparative study. Gastrointest Endosc 2011;74:772-780.

39. Li WB, Zuo XL, Li CQ, et al. Diagnostic value of confocal laser endomicroscopy for gastric superficial cancerous lesions. Gut 2011;60:299-306.

40. Zhang HP, Yang S, Chen WH, Hu TT, Lin J. The diagnostic value of con- focal laser endomicroscopy for gastric cancer and precancerous lesions among Asian population: a system review and meta-analysis. Scand J Gastroenterol 2017;52:382-388.

41. Park JC, Park Y, Kim HK, et al. Probe-based confocal laser endomicroscopy in the margin delineation of early gastric cancer for endoscopic submucosal dissection. J Gastroenterol Hepatol 2017;32:1046-1054.

42. Park CH, Kim H, Jo JH, et al. Role of probe-based confocal laser endomicroscopy-targeted biopsy in the molecular and histopathological study of gastric cancer. J Gastroenterol Hepatol 2019;34:84-91.

43. Kumagai Y, Kawada K, Takubo K, Ishida H. Ultra-high magnification endoscopy (endocytoscopy system) for examination of esophageal lesions. Gastroenterological Endoscopy 2017;59:207-218.

44. Kumagai Y, Takubo K, Kawada K, et al. A newly developed continuous zoom-focus endocytoscope. Endoscopy 2017;49:176-180.

45. Kaise M, Kimura R, Nomura K, et al. Accuracy and concordance of endocytoscopic atypia for the diagnosis of gastric cancer. Endoscopy 2014;46:827-832.

46. Sato H, Inoue $\mathrm{H}$, Ikeda $\mathrm{H}$, et al. In vivo gastric mucosal histopathology using endocytoscopy. World J Gastroenterol 2015;21:5002-5008.

47. Abad MRA, Inoue $\mathrm{H}$, Ikeda $\mathrm{H}$, et al. Utilizing fourth-generation endocytoscopy and the "enlarged nuclear sign" for in vivo diagnosis of early gastric cancer. Endosc Int Open 2019;7:E1002-E1007.

48. Sato H, Inoue H, Hayee B, et al. In vivo histopathology using endocytoscopy for non-neoplastic changes in the gastric mucosa: a prospective pilot study (with video). Gastrointest Endosc 2015;81:875-881.

49. Kutsukawa M, Kudo SE, Ikehara N, et al. Efficiency of endocytoscopy in differentiating types of serrated polyps. Gastrointest Endosc 2014;79:648-656

50. Kimura S, Inoue H, Sato Y, et al. Ex vivo visualization of Helicobacter pylori using an endocytoscopic probe. Biomed Res 2006;27:255-257.

51. Tsurudome I, Miyahara R, Funasaka K, et al. In vivo histological diagnosis for gastric cancer using endocytoscopy. World J Gastroenterol 2017;23:6894-6901. 